\title{
Neutrophil-lymphocyte count ratio better predict prognosis than conventional markers in critical patients? A multiple-centered retrospective cohort study
}

\section{Tao Zhou}

Nanjing Medical University

Nan Zheng

Nanjing Medical University

Xiang Li

Nanjing Medical University

Dongmei Zhu

Nanjing Medical University

Yi Han ( $\square$ hanyi@jsph.org.cn )

The First Affiliated Hospital of Nanjing Medical University

\section{Research article}

Keywords: neutrophil lymphocyte count ratio, procalcitonin, CRP, APACHE I, inflammation, critical illness

Posted Date: November 9th, 2020

DOl: https://doi.org/10.21203/rs.3.rs-102319/v1

License: (c) (i) This work is licensed under a Creative Commons Attribution 4.0 International License. Read Full License

Version of Record: A version of this preprint was published on February 23rd, 2021. See the published version at https://doi.org/10.1186/s12873-021-00418-2. 


\section{Abstract}

Background: Neutrophil-lymphocyte count ratio (NLCR) has been reported as better indicator of bacteremia than procalcitonin (PCT), and better predictor of mortality than C-reactive protein (CRP) in various medical conditions. However, large controversy remains upon this topic. We compared the efficiency of NLCR with conventional inflammatory markers in predicting the prognosis of critical illness.

Methods: We performed a multiple-centered retrospective cohort study consisting of 536 ICU patients with outcomes of survival, 28- and 7-day mortality. NLCR was compared with conventional inflammatory markers such as PCT, C-reactive protein (CRP), serum lactate (LAC), white blood cell, neutrophil and severity score APACHE II (Acute Physiology and Chronic Health Evaluation II) to evaluate the predictive value on outcomes of critical illness. Then receiver operating characteristics (ROC) curves were constructed to assess and compare each marker's sensitivity and specificity respectively.

Results: NLCR values were not differential among survival and mortality groups. Meanwhile remarkable differences were observed upon APACHE II score, CRP, PCT and LAC levels among survival and death groups. ROC analysis revealed that NLCR was not competent to predict prognosis of critical illness. The AUROCs of conventional markers such as CRP, PCT, LAC and APACHE II score were more significant in predicting 28- and 7-day mortality.

Conclusions: NLCR is not competent and less reliable than conventional markers CRP, PCT, LAC and APACHE II score in assessing severity and in predicting outcomes of critical illness.

\section{Background}

Neutrophil lymphocyte count ration (NLCR) has been introduced by Zahorec as a rapid, easy-applicable and cost-effective parameter of inflammation and stress in patients of critical conditions [1]. Until recently, the diagnostic and prognostic value of NLCR on myriad medical situations such as bacteremia [2], sepsis [3], myocardial infarction [4], aneurysmal subarachnoid hemorrhage [5], community acquired pneumonia [6], acute kidney injury [7], liver transplantation [8] and even colorectal cancer, remains a hot topic of investigation [9].

Systemic inflammation is an indispensable process of critical disease, and its severity can be generally associated with the short- and long-term outcomes of critical patients [10]. A great many biomarkers have been applied to assess the severity and progress of systemic inflammation in clinical and research scenarios [10], and to predict the prognosis of various diseases. However, these biomarkers may have limited use due to the lack of sensitivity and specificity as both infection and stress could lead to remarkable changes of these parameters.

Thus, the objective in our current research was to evaluate the association of NLCR with the outcomes of adult critical ill patients, and to assess whether such markers are superior than conventional biomarkers or not. 


\section{Methods}

\section{Data source}

We conducted a retrospective study with data collected from six intensive care units (ICU) in a tertiary university hospital (The First Affiliated Hospital of Nanjing Medical University). Patients included in this database were admitted in these ICUs from Jan 2018 to Jun 2019. All the physiological and pathophysiological data, microbiological results and survival outcomes were recorded accordingly.

\section{Patient records}

Inclusion criteria of the patients: (1) Adults: age of 18 to 89 years; (2) Admitted to ICUs in the First Affiliated Hospital of Nanjing Medical University during the period from Jan 2018 to Jun 2019.

Exclusion criteria of the patients: (1) Hematological disease; (2) Chemotherapy; (3) Receiving glucocorticoids; (4) Receiving bone marrow stimulators. We retrieved the following clinical information for each patient from the database at ICU admission, age, gender, diagnosis, APACHE II score, body temperature, white blood cell count, neutrophil percentage, blood lactate, PCT, CRP, microbiologic results, coexisting diseases and survival records. NLCR was calculated as the ratio of neutrophil and lymphocyte count, as previously described [1].

Patients were divided into three groups according to the survival records: (1) Survival group; (2) 28-day mortality group; (3) 7-day mortality group.

\section{Statistical analysis}

Statistical analysis and graph construction was performed using GraphPad Prism 5.0 and IBM SPSS Statistics 23. Descriptive analysis was conducted for all variables. One way-Anova was applied to evaluate the differences in NLCR, PCT, CRP, LAC levels and APACHE II scores among different groups. Receiver operating characteristic (ROC) curves were built to assess and compare the sensitivity and specificity of the NLCR, PCT, CRP, LAC and APACHE II score in predicting 28-day and 7-day mortality. The area under the ROC curves (AUROCs) varied from 0.5 to 1.0 were accepted, with higher values indicating increased discriminatory ability. Confidence intervals of AUROCs were calculated with non-parametric assumptions. Each biomarker's discriminant ability was compared according to its individual AUROC. For all the comparison in this study, $\mathrm{P}<0.05$ was considered the difference to be statistically significant.

\section{Results}

\section{General characteristics}

Initially 536 patients were enrolled in this study. Following the flow chart with strict inclusion and exclusion criteria, 428 patients were finally analyzed in this study (Figure 1). Of which 310 were medical patients, and 118 were surgical patients. Overall, ICU mortality was $24.5 \%$ (105 of 428 patients), with 
$41.0 \%$ (43 of 105) deaths occurring during the first 7 days after admission, and $59 \%$ (62 of 105) deaths occurring between 7 and 28 days after admission. Table 1 shows the general characteristics of the total enrolled critical ill patients.

Neutrophil count ratio abnormality did not differentiate between survival and mortality groups. Body temperature did not present to have such discriminant potency either. However, the WBC count in 7-day mortality group was much higher than other groups. Surgery patients population in 7-day mortality group presented to be the lowest among all groups, as shown in Table 1, indicating a surgical background may refer a better outcome in critical patients.

Table 1 Characteristics of the overall population

\begin{tabular}{|l|l|l|l|}
\hline & \multicolumn{1}{|c|}{$\begin{array}{c}\text { Survival } \\
\mathrm{n}=\mathbf{3 2 3}\end{array}$} & \multicolumn{1}{|c|}{$\begin{array}{c}\text { 28-day mortality } \\
\mathbf{n}=\mathbf{6 2}\end{array}$} & \multicolumn{1}{|c|}{$\begin{array}{c}\text { 7-day mortality } \\
\mathbf{n}=\mathbf{4 3}\end{array}$} \\
\hline Age (years) & $67.0 \pm 18.7$ & $76.1 \pm 12.7^{* * *}$ & $70.7 \pm 16.9$ \\
\hline Sex (M/F) & $222 / 101$ & $39 / 22$ & $24 / 19$ \\
\hline $\begin{array}{l}\text { Temperature abnormali- } \\
\text { ties, } \mathrm{n},(\%)\end{array}$ & $80(24.8)$ & $21(33.3)$ & $10(23.3)$ \\
\hline WBC abnormalities, $\mathrm{n},(\%)$ & $112(34.7)$ & $24(38.1)$ & $25(58.1)^{* *}$ \\
\hline NE abnormalities, $\mathrm{n},(\%)$ & $227(70.3)$ & $51(82.3)$ & $36(83.7)$ \\
\hline APACHE II score & $18.8 \pm 6.0$ & $24.1 \pm 5.4^{* * *}$ & $24.8 \pm 7.8^{* * *}$ \\
\hline NLCR & $11.5 \pm 13.4$ & $13.0 \pm 11.3$ & $15.0 \pm 14.8$ \\
\hline PCT (ng/ml) & $3.6 \pm 13.8$ & $4.0 \pm 12.5^{* *}$ & $7.8 \pm 15.0^{* * *}$ \\
\hline CRP (mg/ml) & $61.1 \pm 73.1$ & $87.4 \pm 69.1^{* * *}$ & $96.6 \pm 76.0^{* *}$ \\
\hline Blood lactate (mmol/l) & $1.5 \pm 1.0$ & $1.9 \pm 0.9^{* * *}$ & $2.7 \pm 4.0$ \\
\hline Surgery, n, (\%) & $99(30.7)$ & $16(25.8)$ & $3(7.1)^{* * *}$ \\
\hline
\end{tabular}

Data were expressed as number (percentage) of patients or mean \pm standard deviation. ${ }^{* *} p<0.01,{ }^{* * *} p$ $<0.001$ vs survival group. Temperature abnormality: temperature $<36^{\circ} \mathrm{C}$ or $>38^{\circ} \mathrm{C}$; WBC abnormalities: $\mathrm{WBC}<4 \times 10^{\wedge} 9 / \mathrm{L}$ or $>12 \times 10^{\wedge} \mathrm{g} / \mathrm{L}$; NE abnormality: neutrophil count ratio $<40 \%$ or $>75 \%$. 


\section{Microorganisms profile}

Of total analyzed patients, 290 were with evidences of infection, either bacteria (total 300 isolates, with 275 gram-negative and 26 gram-positive isolates), fungi (69 isolates), virus (3 isolates), anaerobe (1 isolate) or tuberculosis (1 isolate). Among all patients, 93 cases (survival: 77 cases, 28-day mortality: 14 cases, 7-day mortality: 6 cases) were with mixed infection with multiple microorganisms, and 148 cases (survival: 116 cases , 28-day mortality: 14 cases, 7-day mortality: 18 cases) were not infected. According to infection sites the patients were with pneumonia (208 cases), bacteremia (19 cases), peritonitis (23 cases), intra-cranial infection (15 cases), and other infections (15 cases). The specific microorganism profile for each group was displayed in table 2 .

Table 2. Microorganisms isolated from the patients in the study cohort 


\begin{tabular}{|c|c|c|c|}
\hline & $\begin{array}{l}\text { Survival } \\
\mathrm{n}=323\end{array}$ & $\begin{array}{l}\text { 28-day mortality } \\
\mathrm{n}=62\end{array}$ & $\begin{array}{l}\text { 7-day mortality } \\
n=43\end{array}$ \\
\hline Gram-Negative isolates (n, \%) & $216(66.9 \%)$ & 39 (62.9\%) & 20 (46.5\%)* \\
\hline Acinetobacter baumannii & $75(23.2 \%)$ & $19(30.6 \%)$ & $13(30.2 \%)$ \\
\hline Klebsiella spp. & $44(13.6 \%)$ & $14(22.6 \%)$ & $3(7.0 \%)$ \\
\hline Pseudomonas spp. & $43(13.3 \%)$ & $5(8.1 \%)$ & $2(4.7 \%)$ \\
\hline Enterobacter spp. & $21(6.5 \%)$ & $1(1.6 \%)$ & $1(2.3 \%)$ \\
\hline S. maltophilia & $14(4.3 \%)$ & $0(0)$ & $1(2.3 \%)$ \\
\hline Other & $19(5.9 \%)$ & $0(0)$ & $0(0)$ \\
\hline Gram-positive isolates (n, \%) & $13(4.0 \%)$ & $9(14.5 \%)^{* *}$ & $4(9.3 \%)$ \\
\hline S.Aureus & $6(1.9 \%)$ & $6(9.7 \%)^{* *}$ & $1(2.3 \%)$ \\
\hline MRSA & $2(0.6 \%)$ & $1(1.6 \%)$ & $1(2.3 \%)$ \\
\hline Streptococcus spp. & $2(0.6 \%)$ & $0(0)$ & $1(2.3 \%)$ \\
\hline Enterococcus spp. & $2(0.6 \%)$ & $1(1.6 \%)$ & $1(2.3 \%)$ \\
\hline Other & $1(0.3 \%)$ & $1(1.6 \%)$ & $0(0)$ \\
\hline Fungi isolates (n, \%) & 52 (16.1\%) & 10 (16.1\%) & 7 (16.3\%) \\
\hline Candida albicans & $35(10.8 \%)$ & $8(12.9 \%)$ & $2(4.7 \%)$ \\
\hline Candida glabrada & $9(2.8 \%)$ & $1(1.6 \%)$ & $1(2.3 \%)$ \\
\hline Candida tropicalis & $5(1.5 \%)$ & $1(1.6 \%)$ & $1(2.3 \%)$ \\
\hline Other & $3(0.9 \%)$ & $0(0)$ & $3(\%) *$ \\
\hline Virus isolates (n, \%) & $2(0.6 \%)$ & $1(1.6 \%)$ & $0(0)$ \\
\hline Anaerobes isolates (n, \%) & $1(0.3 \%)$ & $0(0)$ & $0(0)$ \\
\hline Tuberculosis isolates (n, \%) & $0(0)$ & $0(0)$ & $1(2.3 \%)$ \\
\hline
\end{tabular}

Data were expressed as number (percentage of current group) of isolates, not number of patients. ${ }^{*} \mathrm{p}<$ $0.05,{ }^{*} p<0.01$ vs survival group. Spp., species; S. maltophilia, stenotrophomonas maltophilia; S. aureus, staphylococcus aureus; MRSA, methicillin-resistant staphylococcus aureus.

\section{Co-morbid conditions}


The incidence of cardiovascular co-morbid conditions on admission to the ICU was higher in patients of both 28- and 7-day mortality groups than in survival group, indicating that cardiovascular disease background might be a major risk factor for negative outcomes. On the other hand the incidence of malignancies was much lower in mortality groups than that of survival group. Apart from this, the surgery operation incidence in survival group was much higher than that of 7-day mortality group, as described in Table 1. The general co-morbid disease profile was presented for overall population in Table 3.

Table 3 Coexisting disease of the study population stratified by survival and mortality

\begin{tabular}{|c|c|c|c|}
\hline $\begin{array}{l}\text { 28-day mortality } \\
\mathrm{n}=62\end{array}$ & $\begin{array}{l}\text { Survival } \\
\mathrm{n}=323\end{array}$ & $\begin{array}{l}\text { 28-day mortality } \\
\mathrm{n}=62\end{array}$ & $\begin{array}{l}\text { 7-day mortality } \\
n=43\end{array}$ \\
\hline Diabetes mellitus & $3(0.9 \%)$ & $0(0)$ & $0(0)$ \\
\hline Cardiovascular disease & $18(5.6 \%)$ & $4(6.5 \%)$ & $10(23.3 \%)^{* * *}$ \\
\hline Hypertension & $4(1.2 \%)$ & $0(0)$ & $0(0)$ \\
\hline Malignancies & $29(9.0 \%)$ & $1(1.6 \%)^{* *}$ & $4(9.3 \%)$ \\
\hline COPD & $8(2.5 \%)$ & $4(6.5 \%)$ & $1(2.3 \%)$ \\
\hline Liver cirrhosis & $0(0)$ & $0(0)$ & $0(0)$ \\
\hline Renal failure & $6(1.9 \%)$ & $4(6.5 \%)$ & $2(4.7 \%)$ \\
\hline
\end{tabular}

Data were expressed as number (percentage of current group), ${ }^{* *} \mathrm{p}<0.01, * * * \mathrm{p}<0.001$ vs survival group. COPD, chronic obstructive pulmonary disease.

\section{Diagnostic character of APACHE II and biomarkers}

APACHE II is a severity-of-disease classification system, one of several ICU scoring systems. It is applied within 24 hours of admission of a patient to an ICU. Higher APACHE II score negatively correlates with survival rate. In our study, we applied APACHE II to be the comparable reference for the analyzed biomarkers.

NLCR, APACHE II score and other biomarker levels of survival, 28- and 7-day mortality groups were presented in Figure 2. Considering the total cohort of 428 critical patients, the APACHE II score at admission in mortality groups were much higher than that of survivors. Both CRP and PCT levels of mortality groups were significantly elevated than those of survival groups (Table 1, Figure 2). Regarding to LAC, only 28-day mortality group levels were higher than that of survivals, but not 7-day mortality group levels, probably because of relatively large deviation and small count of group population. Of note, NLCR did not differentiate among survivals and non-survival patients (Table 1, Figure 2).

\section{Discriminant performance of APACHE II and biomarkers}


The discriminant ability of NLCR, CRP, PCT, LAC and APACHE II score to predict 28- and 7-day mortality was presented in Figure 2. With group comparison, we found that PCT, CRP and APACHE II score were all discriminant between survival and mortality groups. While NLCR presented to be not discriminant between groups. Among all biomarkers, both PCT and CRP showed greater differential potency than others, as their $p$ value at comparison of the survival and mortality groups presented to be most significant. On the other hand, LAC showed less differential potency among all groups (Figure 2).

According to area under ROC curves analysis, the AUROCs of NLCR were 0.580 and 0.575 for 28 - and 7day mortality group respectively. And the $P$ values were 0.045 and 0.111 , which did not make NLCR a powerful discriminator to predict mortality. On the other hand, not only APACHE II score, but also CRP and PCT had much higher levels of AUROCs than NLCR, which also made NLCR a relatively weak predictor of mortality (Figure 3).

For discrimination of 28-day mortality, APACHE II score presented highest AUROC than all the biomarkers, while PCT and CRP presented to be less potent biomarkers. LAC had relatively higher levels of AUROC, which made it a good indicator to predict 28-day mortality. For prediction of 7-day mortality, LAC had the lowest value of prediction, meanwhile, APACHE II score remained the highest value of predicting 7-day mortality. Compared to PCT and CRP, NLCR was determined a very weak biomarker to predict both short and long term mortality according to AUROC levels (data shown in Figure 3).

In regard to survive analysis, all the biomarkers selected in this study presented good potential to predict outcomes of critical patients. We calculated the cutoff values for each biomarker and graphed the survival curves according to its values higher or lower than cutoffs. The results showed both PCT and CRP had higher differential ability, with $p$ values lower than 0.001 , better sensitivity and specificity than other markers such as NLCR and LAC. Compared to PCT and CRP, NLCR had presented relatively weak potential to indicate prognosis of critical patients (Figure 4).

\section{Discussion}

Systemic inflammation is an integral part of pathophysiological processes of critical illness. Certain cytokines and some acute phase proteins have been frequently used to assess the inflammatory processes in both clinical and research scenarios. C-reactive protein, white blood cell count and neutrophil percentage have long been recognized as valuable markers of inflammation whether due to infection or not, thus these markers play great roles in recognition of inflammatory status, in assessing the severity of diseases and predicting the following outcomes. However, the sensitivity and specificity is to be determined.

Based on such context, we are constantly in search of a more specific, sensitive, and less costly marker to be able to recognize, to evaluate, and to predict prognosis of critical illness. We evaluated the ability of NLCR, comparing with conventional biomarkers, to predict the prognosis of critical illness, regardless of specific categories of diagnosis and treatments. 
The neutrophil lymphocyte count ratio (NLCR) is a conveniently available marker which could be readily calculated according to complete blood count. Previously NLCR has been proved as a marker of infection, but did not obtain wide acceptance. Countless investigations have demonstrated that NLCR was able to predict outcomes of various oncology patients, and it was also proved to be prognostic pre-operatively in patients with colorectal cancer $[1,11-14]$. Only a few weak quality evidences have presented relationships between NLCR and critical illness, the outcomes and mortality.

Previous investigations had already presented that NLCR was a more sensitive parameter in the prediction of appendicitis [15]. Zahorec and colleagues have observed lymphocytopenia in ICU patients following major surgery and sepsis, and noticed higher levels of NLCR related with severity of the clinical courses [1]. In this study, we demonstrated that NLCR did not have high discriminant ability to predict outcomes of critical illness. Compared with conventional biomarkers and APACHE II score, AUROCs of NLCR did not show advantages of differentiation but just weak predictive capability of 28-day mortality, with relatively low sensitivity and specificity. On the other hand, not only APACHE II score, but also CRP and PCT showed much greater potential of prognostic value on mortality outcomes of critical illness.

Regarding to conventional biomarkers, Wyllie and colleagues have determined that CRP alone could not better predict bacterial infection than lymphocytopenia alone or a combination of lymphocytopenia and neutrophilia [16]. Although procalcitonin has been evaluated and proved to be prognostic with critical illness, especially of septic inflammation, its implementation has been hampered because of its high costs and lacking of accessibility in developing countries.

In our current study, we compared the discriminatory ability of NLCR with CRP, PCT and APACHE II score on outcome prediction of critical illness. The results displayed that PCT, CRP and APACHE II score were all discriminant between survival and mortality group, but NLCR was not differential between survival and mortality group. The ROC analysis also revealed not only APACHE II score, but also CRP, PCT and LAC all had higher levels of AUROCs than NLCR, which made NLCR a relatively weak predictor of mortality. For prediction of 28-day mortality, APACHE II score and LAC presented higher efficacy than all other markers. Meanwhile, for prediction of 7-day mortality, both CRP and PCT displayed comparable efficacy as well as APACHE II score. These results were in accordance with some of previous conclusions [3,17], however in contrary of other investigations [2]. The latter studies may engage in very small population or tumor background, which may likely produce prejudicial clinical conclusions, thus not be able to applied to patients with inflammatory background. Our study enrolled 428 cases into final analysis, which presented a relatively large study population and hence improved its reliability.

Meanwhile, most recent investigations demonstrated that NLCR, a simple and easily obtainable marker, had higher predictive value in bacterial infection, and can be integrated into daily practice without extra costs [2]. Because of these characteristics, NLCR was even suggested to be widely applied to the surveillance protocols of clinical scenarios especially in developing countries. However, in our study, based on AUROCs calculation and comparison, NLCR was proved to be less valuable than any of the above mentioned conventional biomarkers and APACHE II score to predict prognosis or to evaluate the 
severity of a disease. Moreover, NLCR levels did not differentiate between survival and mortality groups, even more suggested it may not capable to be a reliable indicator to evaluate severity or to predict prognosis of critical illness.

\section{Limitations of the study}

Indeed, the present study does have several limitations. First, although this study involves a relatively large population, it is a retrospective study, thus the potential of this study is to be further determined by prospective researches in much larger population in other centers. Second, this study generally compared the potential of NLCR and other traditional inflammatory markers such as CRP, PCT, white blood cell count, neutrophil count and APACHE II severity score, but not the more potentially predictive markers. Further studies should be conducted to evaluate such predictive potentials and compare them with those short- and long-term bio-reactive proteins, such as acute phase proteins in this context. Third, this study did not separately demonstrate relationships between NLCR and risk of death in infected patients. At this point, it may contradict with the conclusions of other literatures.

\section{Conclusions}

The NLCR, CRP and PCT were associated with both 7- and 28-day mortality in adult critical patients. Both PCT and CRP were more sensitive and specific to predict prognosis in critical ill patients. Compared to traditional predictive indicators, NLCR has no advantages over PCT, CRP, and APACHE II score. Thus, NLCR could not be an ideal substitute to conventional markers in evaluation of severity of critical illness.

\section{List Of Abbreviations}

APACHE II: Acute Physiology and Chronic Health Evaluation II; AUROC: Area under the ROC curve; Cl: confidence interval; CRP: C-reactive protein; ICU: intensive care unit; IRB: institutional review boards; LAC: lactate; NE: neutrophil; NLCR: neutrophil-lymphocyte count ratio; PCT: procalcitonin; ROC: receiver operating characteristic; WBC: white blood cell.

\section{Declarations}

\section{Ethics approval and consent to participate}

We have received ethical approval (2020-SR-055) from the institutional review boards (IRBs) at the First Affiliated Hospital of Nanjing Medical University. Since this study does not contain protected health information and all data were anonymously used, a waiver of the requirement for informed consent was approved by the IRBs. Individual patients consent was not obtained since all data used in this study were acquired retrospectively from the laboratory information system without any additional sampling or laboratory analysis.

\section{Consent for publication}


Yes. We the authors all give consent for publication.

\section{Availability of data and materials}

The data-sets used and/or analyzed during the current study are available from the corresponding author on reasonable request.

\section{Competing interests}

The authors declare that they have no competing interests.

\section{Funding}

This study was financed by National Natural Science Foundation of China, No.81770287.

\section{Authors' contributions}

TZ, DZ and XL retrieved the data of patients according to the background of critical illness, survival and mortality. YH analyzed and interpreted the data, then constructed the manuscript. All authors read and approved the final manuscript.

\section{Acknowledgments}

The statistical analysis of this study received help from Dr. Qiulun Lu and Dr. Yifeng Wang from Nanjing Medical University, Nanjing, China.

\section{Authors' information}

Department of Critical Care Medicine, the First Affiliated Hospital of Nanjing Medical University, Nanjing Medical University, China, 210029.

\section{References}

1. Zahorec R. Ratio of neutrophil to lymphocyte counts-rapid and simple parameter of systemic inflammation and stress in critically ill. Bratisl Lek Listy. 2001;102(1):5-14.

2. de Jager CP, van Wijk PT, Mathoera RB, de Jongh-Leuvenink J, van der Poll T, Wever PC. Lymphocytopenia and neutrophil-lymphocyte count ratio predict bacteremia better than conventional infection markers in an emergency care unit. Crit Care. 2010;14(5):R192.

3. Westerdijk K, Simons KS, Zegers M, Wever PC, Pickkers P, de Jager CPC. The value of the neutrophillymphocyte count ratio in the diagnosis of sepsis in patients admitted to the Intensive Care Unit: A retrospective cohort study. PLoS One. 2019;14(2):e0212861.

4. Hong D, Choi KH, Song YB, Lee JM, Park TK, Yang JH, Hahn JY, Choi JH, Choi SH, Kim SM, Choe Y, Kim EK, Chang SA, Lee SC, Oh JK, Gwon HC. Prognostic implications of post-percutaneous coronary 
intervention neutrophil-to-lymphocyte ratio on infarct size and clinical outcomes in patients with acute myocardial infarction. Sci Rep. 2019;9(1):9646.

5. Giede-Jeppe A, Reichl J, Sprügel MI, Lücking H, Hoelter P, Eyüpoglu IY, Kuramatsu JB, Huttner HB, Gerner ST. Neutrophil-to-lymphocyte ratio as an independent predictor for unfavorable functional outcome in aneurysmal subarachnoid hemorrhage. J Neurosurg. 2019:1-8.

6. Curbelo J, Rajas O, Arnalich B, Galván-Román JM, Luquero-Bueno S, Ortega-Gómez M, Lancho A, Roy E, Sánchez Azofra A, Mateo Jiménez G, Gómez M, Moldenhauer F, Aspa J. Neutrophil Count Percentage and Neutrophil-Lymphocyte Ratio as Prognostic Markers in Patients Hospitalized for Community-Acquired Pneumonia. Arch Bronconeumol. 2019;55(9):472-7.

7. Zhou X, Liu J, Ji X, Yang X, Duan M. Predictive value of inflammatory markers for acute kidney injury in sepsis patients: analysis of 753 cases in 7 years. Zhonghua Wei Zhong Bing Ji Jiu Yi Xue. 2018;30(4):346-50.

8. Kwon HM, Moon YJ, Jung KW, Park YS, Jun IG, Kim SO, Song JG, Hwang GS. Neutrophil-tolymphocyte ratio is a predictor of early graft dysfunction following living donor liver transplantation. Liver Int. 2019;39(8):1545-56.

9. Povsic MK, Beovic B, Ihan A. Perioperative Increase in Neutrophil CD64 Expression is an Indicator for Intra-abdominal Infection after Colorectal Cancer Surgery. Radiol Oncol. 2016;51(2):211-20.

10. Cheval C, Timsit JF, Garrouste-Orgeas M, Assicot M, De Jonghe B, Misset B, Bohuon C, Carlet J. Procalcitonin (PCT) is useful in predicting the bacterial origin of an acute circulatory failure in critically ill patients. Intensive Care Med. 2000;26 Suppl 2:S153-8.

11. Sarraf KM, Belcher E, Raevsky E, Nicholson AG, Goldstraw P, Lim E. Neutrophil / lymphocyte ratio and its association with survival after complete resection in non-small cell lung cancer. J Thorac Cardiovasc Surg. 2009;137(2):425-8.

12. Cho H, Hur HW, Kim SW, Kim SH, Kim JH, Kim YT, Lee K. Pre-treatment neutrophil to lymphocyte ratio is elevated in epithelial ovarian cancer and predicts survival after treatment. Cancer Immunol Immunother. 2009;58(1):15-23.

13. Azab B, Bhatt VR, Phookan J, Murukutla S, Kohn N, Terjanian T, Widmann WD. Usefulness of the neutrophil-to-lymphocyte ratio in predicting short- and long-term mortality in breast cancer patients. Ann Surg Oncol. 2012;19(1):217-24.

14. Walsh SR, Cook EJ, Goulder F, Justin TA, Keeling NJ. Neutrophil-lymphocyte ratio as a prognostic factor in colorectal cancer. J Surg Oncol. 2005;91(3):181-4.

15. Goodman DA, Goodman CB, Monk JS. Use of the neutrophil:lymphocyte ratio in the diagnosis of appendicitis. Am Surg. 1995;61(3):257-9.

16. Wyllie DH, Bowler IC, Peto TE. Bacteraemia prediction in emergency medical admissions: role of C reactive protein. J Clin Pathol. 2005;58(4):352-6.

17. Riché F, Gayat E, Barthélémy R, Le Dorze M, Matéo J, Payen D. Reversal of neutrophil-to-lymphocyte count ratio in early versus late death from septic shock. Crit Care. 2015;19:439. 
Figures

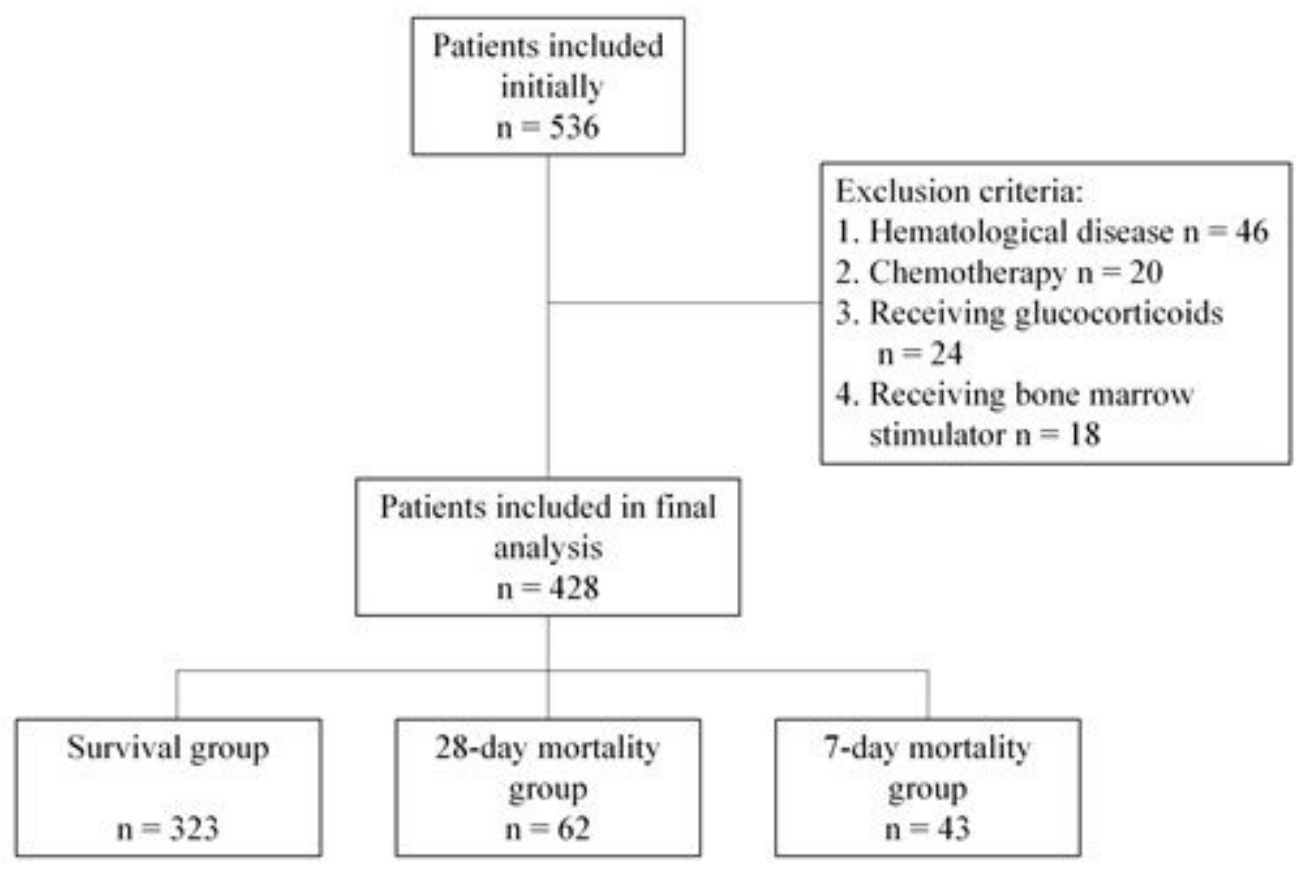

\section{Figure 1}

Enrollment flow chart. 

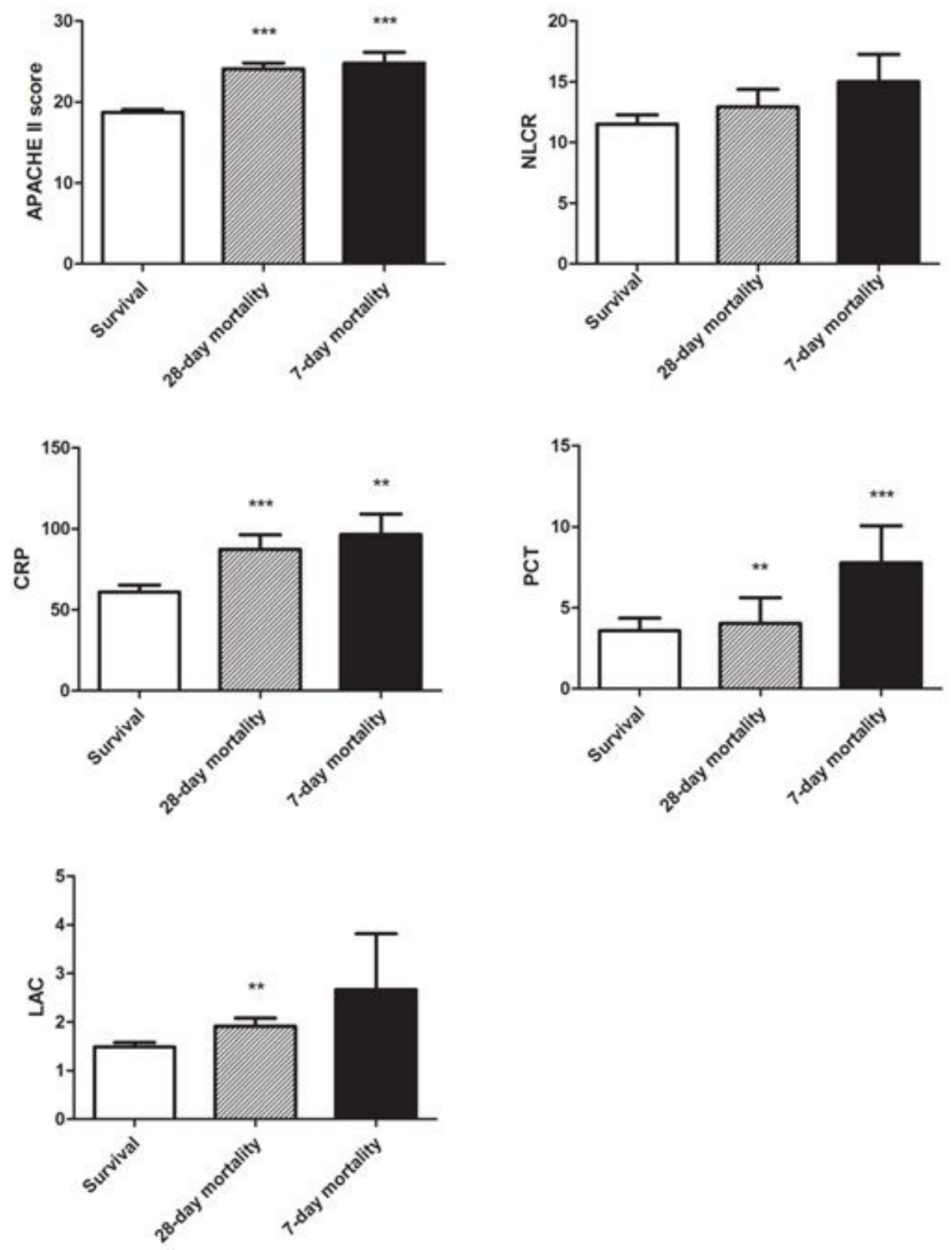

Figure 2

APACHE II scores and biomarker levels of NLCR, CRP, PCT, and LAC of survival, 28-day mortality, and 7day mortality group. ${ }^{* *} \mathrm{p}<0.01$ vs survival, ${ }^{* *} \mathrm{p}<0.001$ vs survival. 
28-day mortality

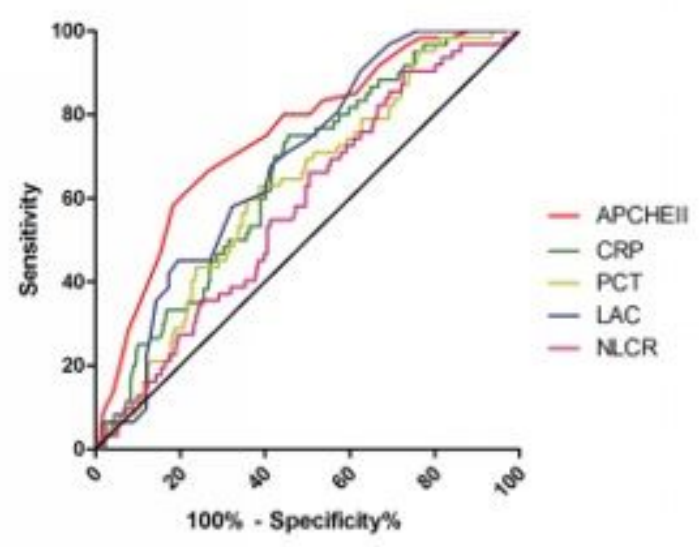

28-day mortality

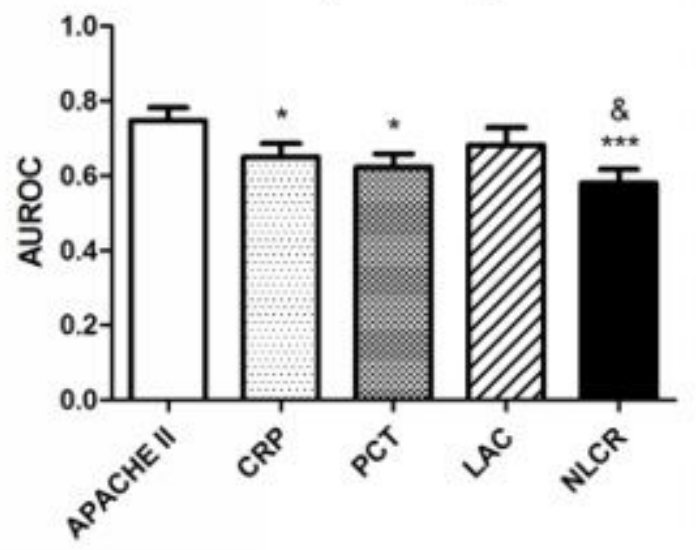

7-day mortality

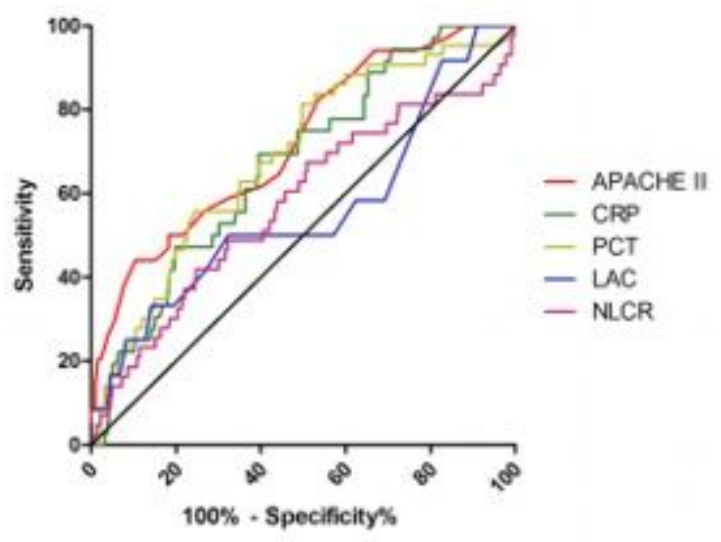

7-day mortality

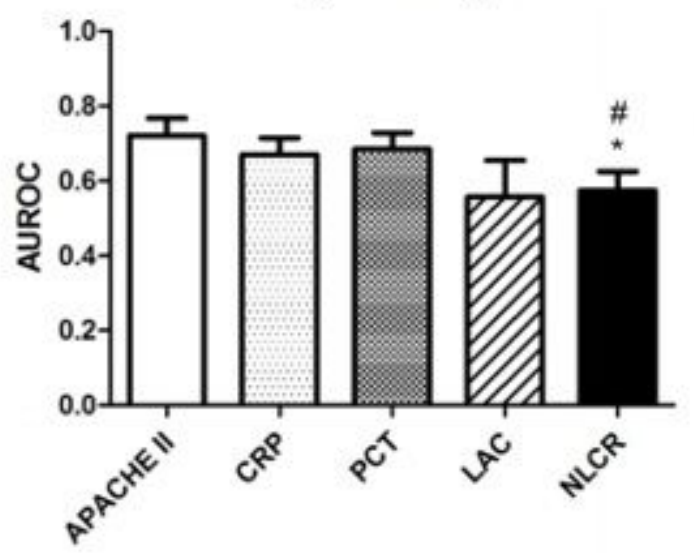

\begin{tabular}{|l|r|r|r|r|r|r|r|r|r|r|}
\hline & \multicolumn{4}{|c|}{ 28-day mortality } & \multicolumn{5}{|c|}{ 7-day mortality } \\
\hline & APACHE II & CRP & PCT & LAC & NLCR & APACHE II & CRP & PCT & LAC & NLCR \\
\hline AUROC & 0.748 & 0.651 & 0.623 & 0.682 & 0.580 & 0.721 & 0.671 & 0.686 & 0.557 & 0.575 \\
\hline STD & 0.034 & 0.035 & 0.036 & 0.047 & 0.037 & 0.047 & 0.044 & 0.043 & 0.097 & 0.051 \\
\hline CI(95\%) lower & 0.683 & 0.581 & 0.552 & 0.591 & 0.508 & 0.630 & 0.584 & 0.602 & 0.366 & 0.475 \\
\hline CI(95\%) upper & 0.814 & 0.720 & 0.693 & 0.774 & 0.652 & 0.812 & 0.757 & 0.769 & 0.748 & 0.674 \\
\hline P value & $\mathbf{0 . 0 0 1}$ & $<\mathbf{0 . 0 0 1}$ & $\mathbf{0 . 0 0 2}$ & $\mathbf{0 . 0 0 2}$ & $\mathbf{0 . 0 4 5}$ & $<\mathbf{0 . 0 0 1}$ & $-\mathbf{0 . 0 0 1}$ & $<\mathbf{0 . 0 0 1}$ & 0.514 & 0.111 \\
\hline
\end{tabular}

\section{Figure 3}

Receiver operating characteristic curves and AUROCs of markers for predicting overall 28-day and 7-day mortality. ${ }^{*}<<0.05, * \star * P<0.001$ compared with APACHE II, \&P $<0.05$ compared with LAC, \#P $<0.05$ compared with PCT. The table below showed the area under the ROC curve value with STD, 95\% confidence intervals and $P$ values versus survival group. 
NLCR
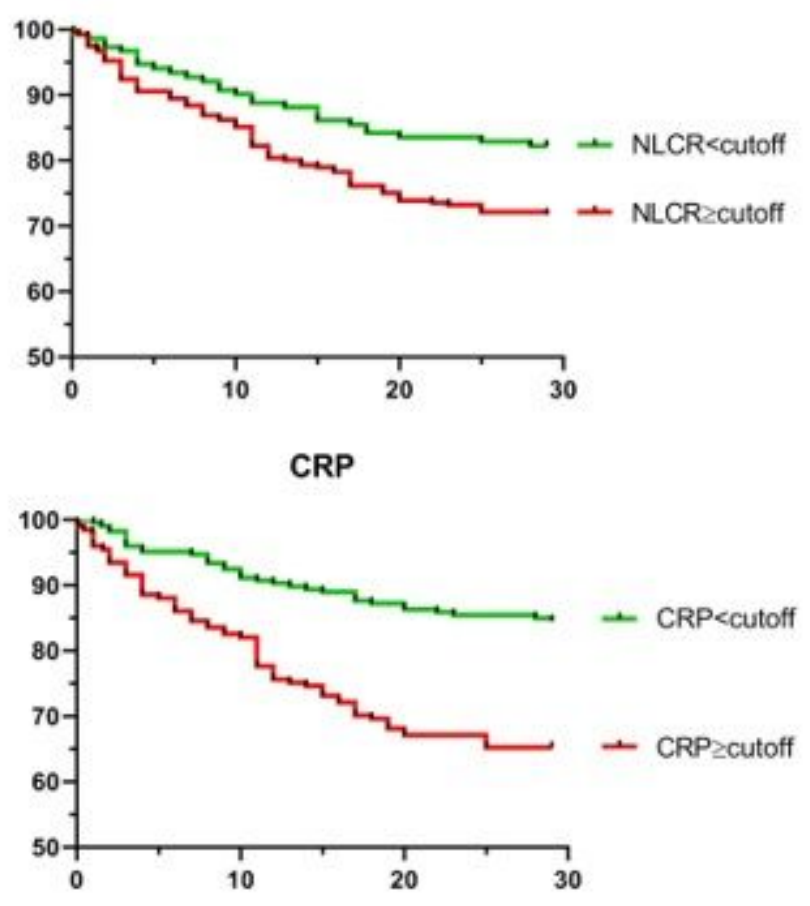

PCT

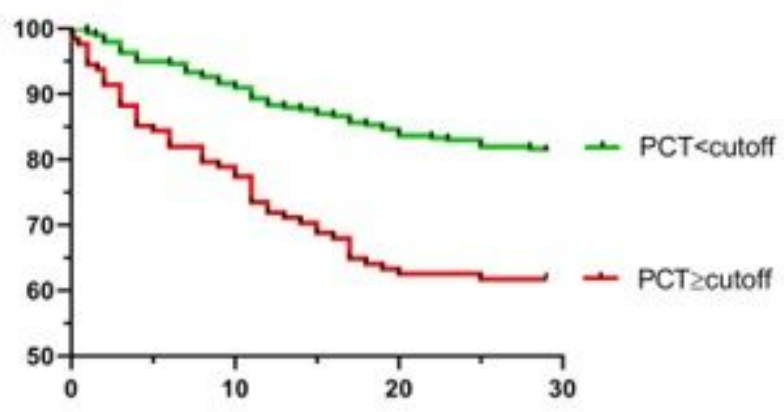

LAC

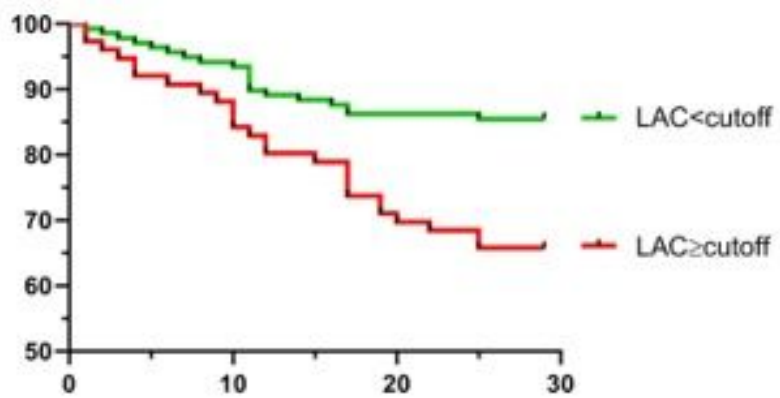

\begin{tabular}{|l|l|l|l|l|}
\hline & $P$ value & Cutoff value & Sensitivity(\%) & Specificity(\%) \\
\hline NLCR & 0.013 & 7.2 & 66.7 & 49.5 \\
\hline PCT & $<0.001$ & 0.54 & 64.8 & 59.8 \\
\hline CRP & $<0.001$ & 44.15 & 73.3 & 55.7 \\
\hline LAC & 0.003 & 1.69 & 56.5 & 70.2 \\
\hline
\end{tabular}

\section{Figure 4}

Survival curves of biomarkers divided by respective cutoff values. The table below showed the P values, cutoff values, sensitivity and specificity. 